\title{
Development and in vivo Evaluation of Hydroxy- $\alpha$-Sanshool Intranasal Liposomes as a Potential Remedial Treatment for Alzheimer's Disease
}

\author{
Ruolan Li* \\ Feng Lu* \\ Xue Sun (DD \\ Liying $\mathrm{He}$ \\ HuXinyue Duan \\ Wei Peng \\ Chunjie Wu (1D)
}

State Key Laboratory of Southwestern Chinese Medicine Resources, School of Pharmacy, Chengdu University of Traditional Chinese Medicine, Chengdu, 6I II37, People's Republic of China

*These authors contributed equally to this work
Correspondence: Wei Peng; Chunjie Wu $\mathrm{Tel} / \mathrm{Fax}+86-28-6 \mathrm{I} 80 \mathrm{I} 00 \mathrm{I}$

Email pengwei@cdutcm.edu.cn; wucjcdtcm@I63.com
Purpose: Hydroxy- $\alpha$-sanshool (HAS) improves cognitive dysfunction, but its structural instability has limited its clinical application. The present study was conducted to investigate the optimal formulation of hydroxy- $\alpha$-sanshool liposomes (HAS-LPs) and its effect on ameliorating learning and memory disorders in an Alzheimer's disease (AD) model.

Methods: In this study, HAS was prepared as HAS-LP using a thin film dispersion method. After selecting the optimal preparation conditions, HAS-LP was characterized using transmission electron microscopy (TEM) and by measuring the zeta potential, particle size, and in vitro drug release. Next, evaluated the effect of HAS-LP on the rat nasal mucosa and then applied it to $\mathrm{AD}$ mice. By performing behaviour experiments, pathological test and related pharmacokinetic parameters, we explored its effect on attenuating learning and memory impairment in mice. Results: When the mass ratio of HAS:cholesterol:soybean lecithin was 1:4:16 and $15 \mathrm{~mL}$ of ultrapure water were added, the highest encapsulation efficiency and drug loading were obtained. HAS-LP had a particle size of $181.77 \mathrm{~nm}$, a polydispersity index of 0.207 and a zeta potential of $-53.8 \mathrm{mV}$, and it remained stable at $25{ }^{\circ} \mathrm{C}$ for 1 week and $4{ }^{\circ} \mathrm{C}$ for 8 weeks. Moreover, HAS-LP exhibited slow drug release and was highly consistent with the Higuchi release model. HAS-LP was not significantly toxic to the nasal mucosa and effectively alleviated D-galactose-induced learning memory deficits and protected mouse hippocampal neuronal cells. HAS-LP was highly enriched in plasma and brain tissue after administration via the nasal route and obtained some ability to target the brain.

Conclusion: HAS encapsulated in soybean lecithin and cholesterol was successfully developed, suggesting that treatment with the nanoparticles might reverse some AD symptoms. Therefore, these nanoparticles might be used as promising new candidates for the delivery of HAS to treat AD.

Keywords: hydroxy- $\alpha$-sanshool, intranasal liposome, Alzheimer's disease

\section{Introduction}

As the ageing population increases and due to progressive nature of disease, Alzheimer's disease (AD) has become one of the most common diseases in the elderly population and is a serious threat to the lives of the elderly. ${ }^{1} \mathrm{AD}$ is a progressive neurodegenerative disease and its onset is insidious, long, and irreversible. A previous study suggested that the clinical symptoms of $\mathrm{AD}$ are divided into memory and cognitive impairments, accompanied by reduced self-care ability and abnormal mental manifestations while its aetiology and pathogenesis are still not fully understood. ${ }^{2,3}$ Furthermore, the pathological features of $\mathrm{AD}$ are the presence of senile plaques and neurofibrillary tangles in the brain, 
while its proposed pathogenic mechanisms mainly include cholinergic dysfunction, the tau protein hyperphosphorylation hypothesis, the $A \beta$ cascade hypothesis and the oxidative stress hypothesis. ${ }^{46}$ Nevertheless, the current drug pipeline for AD in clinical trials remains dry, as cholinesterase inhibitors and glutamate receptor antagonists only ameliorate symptoms and do not cure the disease. ${ }^{7}$ In addition, which is the most fragile and important organ in the human body, has a blood-brain barrier (BBB) composed of capillary endothelial cells that are closely apposed by tight intercellular junctions that lack fenestrations and have high transendothelial electrical resistance, thereby limiting the paracellular diffusion of solutes or drugs. 8,9

Intranasal drug delivery, a noninvasive drug administration approach, is a traditional treatment that is mainly applied to local nasal diseases. Interestingly, this traditional treatment has attracted increasing interest in recent years since it directly delivers a drug to the cerebrospinal fluid which can not only maintains the integrity of the drug but also achieves targeting, circumventing the BBB. ${ }^{10}$ Therefore, nasal administration of treatments for neurodegenerative diseases has also received increasing attention. ${ }^{11}$ For example, after nasal administration, drugs exert their effects by stimulating the olfactory nerve, facial nerve and the ocular and maxillary branches of the trigeminal nerve in the nasal cavity. ${ }^{12}$ However, different results have also been reported for the time and content of different drugs absorbed into the brain after nasal administration. According previous related studies, nondissociated fatsoluble drugs with a molecular weight of less than 1000 are more easily absorbed through the nasal cavity and factors such as the nasal mucociliary system and enzymes will also directly affect the absorption and distribution of the drug. ${ }^{13}$

Undoubtedly, herbal medicines are beneficial for treating various diseases due to their low toxicity and side effects. Zanthoxylum bungeanum, belonging to the Rutaceae family, is a known medicinal plant that is widely distributed in China. ${ }^{14}$ In addition, modern pharmacological and phytochemical evidence has shown that the essential oil of in Z. bungeanum pericarps exerts a variety of pharmacological effects, including antitumour effects, anti-inflammatory effects, and antibacterial and insecticidal activities. ${ }^{15,16}$ Interestingly, our previous work showed that a unsaturated fatty acid amide from $Z$. bungeanum, hydroxy- $\alpha$-sanshool (HAS), protects PC12 cells from oxidative stress induced by $\mathrm{H}_{2} \mathrm{O}_{2}$ stimulation, thereby inhibiting apoptosis. Namely, HAS represents a potential treatment for AD. ${ }^{17}$ However, HAS is extremely unstable under conditions of room temperature, light, oxygen-containing air, and ultraviolet light and is converted into its isomers. Therefore, in our experiments, the stability of HAS was expected to be improved by encapsulating HAS in liposomal materials and isolating HAS from air, UV light, and other stimuli. Additionally, the optimal process for preparing HAS-LP was determined to obtain in a higher encapsulation rate, drug loading capacity and physicochemical characteristics and to transport more drugs into the body following nasal administration. Subsequently, the prepared HAS-LP was administered through the nose to provide HAS-LP a greater ability to pass through the BBB and hopefully to achieve better efficacy in the treatment of $\mathrm{AD} .^{18}$

\section{Experiment Materials, Reagents and Animals}

Hydroxy- $\alpha$-sanshool (HAS) (purity greater than 98\%) used in the present study was isolated from the Z. bungeanum pericarps and supplied by PUSH Bio-Technology (Chengdu, China). Soybean lecithin (98\% phosphatidylcholine, PC), cholesterol, sodium deoxycholate and normal saline were purchased from Shanghai Rhawn Chemical Technology Co., Ltd (Shanghai, China). HPLC-grade acetonitrile and methanol were obtained from Thermo Fisher Scientific (Massachusetts, USA) while phosphate-buffered saline (PBS) was provided by Gibco Co. (Grand Island, NY, USA). Methyl alcohol, trichloromethane and diethyl ether (analytical grade) were purchased from Kelong Chemical Co., Ltd (Chengdu, China). Dialysis tubes were obtained from Viskase Co., Inc (Lombard Illinois, USA). 10\% neutral formaldehyde fixation solution and eosin were provided by Baso Biotechnology Co., Ltd (Zhuhai, China). Sodium dihydrogen phosphate and disodium hydrogen phosphate were obtained from Fuchen Chemical Reagent Co., Ltd (Tianjin, China). Haematoxylin was purchased from Servicebio Biotechnology Co., Ltd (Wuhan, China). All other reagents used in the experiments were of analytical grade. All animals were purchased from Dashuo Experimental Animal Co., Ltd and were conducted in accordance with the National Institutes of Health Guide for the Care and Use of Laboratory Animals, and approved by the Animal Care and Use Committee of Chengdu University of Traditional Chinese Medicine. The ethical approval number for conducting the in vivo study in animals was $201,920$.

\section{HPLC Assay of HAS}

HAS was analysed using to the method described in previous report. ${ }^{19}$ HPLC analysis was performed using an 
Agilent 1260 liquid chromatography system with a UV visible detector (Santa Clara, CA, USA). For all HPLC analyses, a Wondasil $\mathrm{C}_{18}$ chromatography column $(5 \mu \mathrm{m}$, $4.6 \times 250 \mathrm{~mm}$; Shimadzu, Japan) was used. The mobile phase consisted of acetonitrile/ultrapure water (v/v, 40:60) at a flow rate of $1 \mathrm{~mL} / \mathrm{min}$ and the detection wavelength was set at $270 \mathrm{~nm}$, while the column temperature was maintained at $30{ }^{\circ} \mathrm{C}$. Samples of $10 \mu \mathrm{L}$ were injected and a calibration curve was prepared with a $R^{2}=0.999$. The HAS content was calculated from the standard curve. As shown in Figure 1, the selected chromatographic conditions ensured that the peak time of the blank liposome material differed substantially from that of HAS and the peak shape of HAS conformed to the determination regulations, which were used to determination of HAS content.

\section{Screening the Preparation of HAS Nano-Liposomes (HAS-LPs)}

The thin-film dispersion method was selected to prepare HAS-LPs after the preliminary experimental screen, and a $3^{3}$ factorial design was selected based on the previous single-factor analysis to further explore the effects of three variables on HAS-LP preparation. In factorial experiments, the differences between multiple levels of all factors can be comprehensively studied. The research variables are the mass ratio of HAS to lipid material $(1: 15,1: 20$ or $1: 25)$, the mass ratio of cholesterol to soy lecithin $(1: 3,1: 4$ or $1: 5)$ and the volume of ultrapure water $(15,20$ or $25 \mathrm{~mL})$. The preparation process is briefly summarized below. First, $3 \mathrm{mg}$ of HAS and lipid materials of different weights were weighed and mixed with $10 \mathrm{~mL}$ of trichloromethane to dissolve HAS and lipid materials. Subsequently, a rotary evaporator was used to evaporate the solvent and form a thin film. Different volumes of ultrapure water were added after vacuum drying, hydrated in an ice water bath and ultrasonicated for $20 \mathrm{~min}$ to completely dissolve the film in ultrapure water and form a uniform and stable mixture. Finally, the prepared HAS-LP solution was filtered three times through a $200 \mathrm{~nm}$ filter membrane to obtain different formulations of HAS-LPs. The formulations are depicted in Table 1.

Encapsulation efficiency and loading capacity were determined using high-performance liquid chromatography (HPLC) method after dialysis. Then, we selected one treatment for subsequent studies according to the results for the encapsulation efficiency, loading capacity, average particle size, PDI and zeta potential. The encapsulation efficiency (\%) and loading capacity (\%) of HAS in HASLP were calculated using the following equations:

$$
\begin{gathered}
\text { Encapsulation efficiency }(\%)=\left(1-\frac{W a}{W b}\right) \times 100 \% \\
\text { Loading capacity }(\%)=\frac{W b-W a}{W b+W c} \times 100 \%
\end{gathered}
$$

where, $W a$ represents the amount of unentrapped HAS in the dialysate, $W b$ is the total amount of HAS in the HASLP, and $W c$ represents the amounts of excipients (soybean lecithin and cholesterol) that were used in the preparation.

\section{Characterization, Storage Stability and Release Rate in vitro}

HAS-LP was characterized by measuring the particle size, particle size distribution (reported as polydispersity index, or PDI) and the Zeta charge ( $\zeta$, potential). These properties were determined using a transmission electron microscope and Zetasizer Nano S90 dynamic light scattering device. For Zeta potential measurements, the HAS-LP was diluted

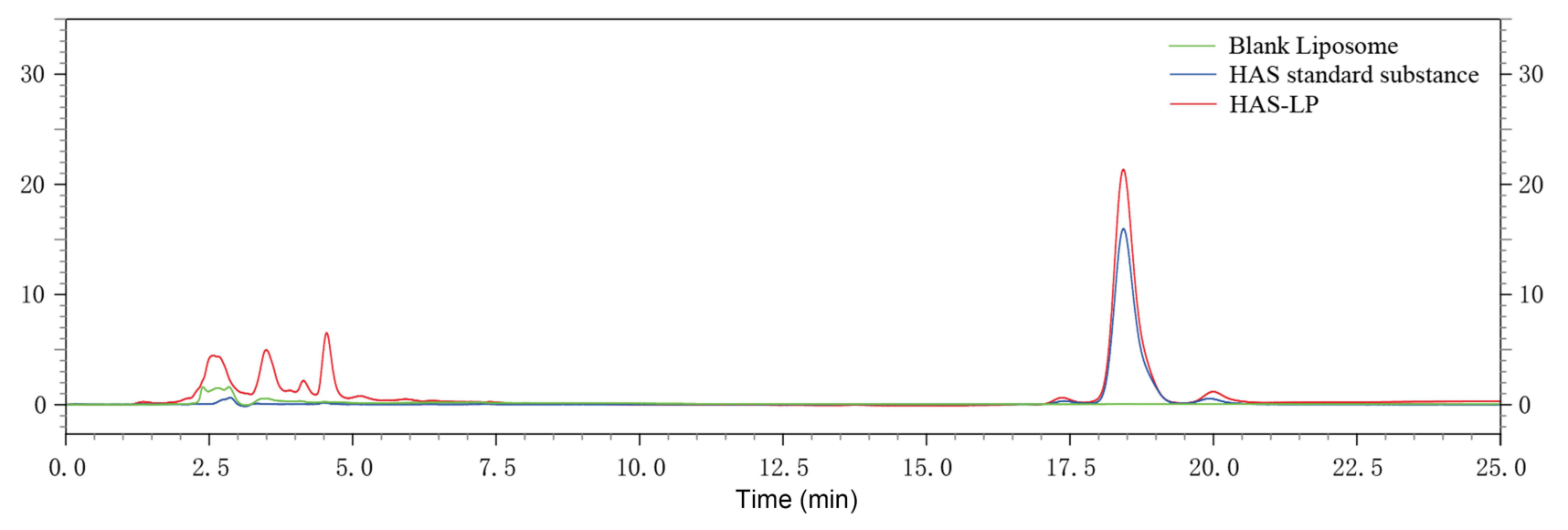

Figure I HPLC analysis. 
Table I Screening the Preparation of Hydroxy- $\alpha$-Sanshool Nano-Liposomes

\begin{tabular}{|l|c|c|c|}
\hline Treatment & $\begin{array}{c}\text { HAS : Lipid Material } \\
\text { (Mass Ratio) }\end{array}$ & $\begin{array}{c}\text { Cholesterol : Soy Lecithin } \\
\text { (Mass Ratio) }\end{array}$ & Ultrapure Water (mL) \\
\hline 1 & $1: 15$ & $1: 3$ & 15 \\
2 & $1: 20$ & $1: 4$ & 15 \\
3 & $1: 25$ & $1: 5$ & 15 \\
4 & $1: 25$ & $1: 4$ & 20 \\
5 & $1: 20$ & $1: 3$ & 20 \\
6 & $1: 15$ & $1: 5$ & 20 \\
9 & $1: 15$ & $1: 4$ & 25 \\
9 & $1: 20$ & $1: 3$ & 25 \\
\end{tabular}

$1: 20$ in deionized microfiltered water and the average surface charge was determined. All measurements were performed in triplicate at $25{ }^{\circ} \mathrm{C}$. Meanwhile, the storage stability of HAS and HAS-LP was determined at $25^{\circ} \mathrm{C}$ for 1 week and $4{ }^{\circ} \mathrm{C}$ for 8 weeks.

PBS $\left(37^{\circ} \mathrm{C}\right)$ was used as the dissolution medium to investigate drug release from HAS-LP in vitro. One millilitre of HAS-LP into the dialysis bag, perform dynamic dialyzing at $80 \mathrm{r} / \mathrm{min}$ and $2 \mathrm{~mL}$ of the dialysate were removed at each time point $(0,1,2,3,4,5,6,7$, and 8 h). The HAS content was determined, cumulative release of HAS-LP was calculated, and Origin 9.0 software was used to apply zero-order kinetics, first-order kinetics, and Higuchi models for fitting. Meanwhile, the in vitro release of HAS was used as a control group for the study.

\section{Nasal Mucosa Toxicity Study}

Rats $(200 \pm 20 \mathrm{~g})$ were purchased from Dashuo Experimental Animal Co., Ltd and housed under suitable temperature $\left(22 \pm 2^{\circ} \mathrm{C}\right)$ and humidity $(65 \pm 5 \%)$ in the animal observation room of Chengdu University of Traditional Chinese Medicine with free access to water and food. All animal experiments were performed according to the National Institutes of Health Guide for the Care and Use of Laboratory Animals. Rats were randomly divided into four groups ( $\mathrm{n}=5$ in each group): normal saline (control group), 1\% deoxysodium cholate group, HAS group and HAS-LP group. After adaptive feeding for 5 days, use a pipette to instill the corresponding drugs into the nasal cavity $(50 \mu \mathrm{L}$ for the left and right nasal cavities) for 7 days. On the 8th day, the rats were euthanized and the nasal mucosa of the rats was removed and stained with H\&E for observation and image acquisition.

\section{Behavioral Evaluation}

The mice $(20 \pm 2 \mathrm{~g})$ were purchased from Dashuo Experimental Animal Co., Ltd and housed under suitable temperature $\left(22 \pm 2{ }^{\circ} \mathrm{C}\right)$ and humidity $(65 \pm 5 \%)$ in the animal observation room of Chengdu University of Traditional Chinese Medicine with free access to water and food. The mice were randomly divided into six groups ( $\mathrm{n}=8$ in each group): the normal group (normal saline), the control group (D-gal), the positive group (donepezil) and the three treatment groups $(1.25,2.5$, and $5.0 \mathrm{mg} / \mathrm{kg}$ HAS). D-gal was dissolved in sterile saline while donepezil was dissolved in $0.5 \% \mathrm{CMC}-\mathrm{Na}$ for administration. After adaptive feeding for 5 days, mice in the normal group and control group were intranasally administered an equal volume of normal saline, mice in the positive group were orally administered $2.0 \mathrm{mg} / \mathrm{kg}$ donepezil, and mice in the treatment groups were administered 1.25, 2.5 and. $5.0 \mathrm{mg} / \mathrm{kg}$ of HAS-LP by nasal drip, respectively. Afterwards, except for the normal group, each group of mice was subcutaneously injected with $500 \mathrm{mg} / \mathrm{kg}$ of D-gal once a day for 50 days. The experimental flowchart is shown in Figure 2.

The passive avoidance test and the Morris water maze test were conducted on the 42nd and 47th day of the experiment, respectively. All tests were conducted under dim light and low noise conditions. The passive avoidance test lasted for two days, the first day was the training trial and the second day was the test trial. The passive avoidance test apparatus was equipped with identical illuminated and unilluminated compartments with an electrifiable copper plate in an unilluminated compartment. The two compartments were connected by a hole. On the first day of this experiment, the mice were placed 


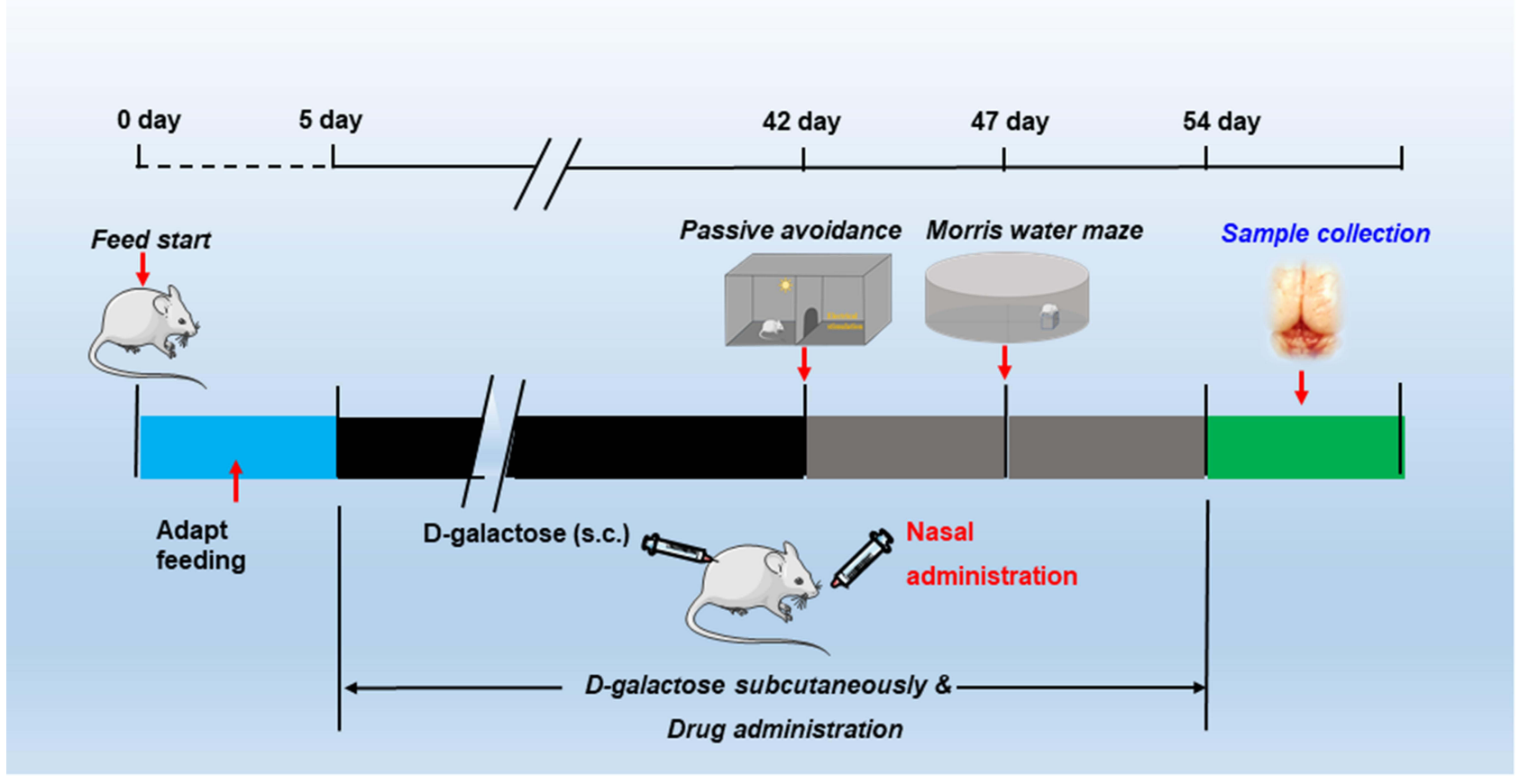

Figure 2 Flowchart of animal experiment.

in illuminated compartment for 2 minutes to adapt to the environment, and then the hole were opened. After the mouse entered the dark room, the hole was closed and the stimulator is turned on to pass current. Twenty-four hours after the training trial, we performed a test trial and recorded the time when the mouse received electrical stimulation for the first time (recorded as "Escape latency") and the number of times the mouse received electrical stimulation within $200 \mathrm{~s}$ (recorded as "The number of errors"). If the mouse received no electrical stimulation within $200 \mathrm{~s}$, the time was recorded as $200 \mathrm{~s}$.

In the Morris water maze test, the main device was a pool with a diameter of $110 \mathrm{~cm}$ and a height of $50 \mathrm{~cm}$, in which black ink was dispersed and a platform was placed in the second quadrant. During the experiment, the water temperature in the pool was maintained at $20^{\circ} \mathrm{C}$. On the 47 th day of the experiment, mice were placed in the pool at random locations, and the time required to find the platform was recorded. If the mice failed to find the platform within $60 \mathrm{~s}, \mathrm{t}$ the investigator held it to on the platform for $20 \mathrm{~s}$ and the result was recorded as "Escape latency". This stage lasted for 5 days. On the 52nd day, the invisible platform was removed from the pool. The time that the mouse remained in the second quadrant and the number of times it crossed the platform location in the second quadrant were recorded.

\section{Histological Analysis}

On the 54th day of the experiment, the mice were sacrificed and the brain tissues from 5 mice in each group were collected and fixed with $4 \%$ paraformaldehyde. After embedding and staining with haematoxylin and eosin, a digital slice scanner was used to observe the slices at $40 \times$ and $400 \times$ magnification, and the images were collected at the same time to score the damage according to the different proportions of neuronal damage. The scoring criteria are shown in Table 2. After Nissl staining and TUNEL staining, the number of Nissl bodies and apoptotic cells were observed using the same method.

\section{Pharmacokinetic Study}

In vivo pharmacokinetic studies were performed in mice with $\mathrm{AD}$ after modelling to evaluate the bioavailability of

Table 2 Neuronal Injury Scoring Standard

\begin{tabular}{|l|c|}
\hline Score & Diagnostic Criteria (Percentage of Damage) \\
\hline 0 & 0 \\
0.5 & $<10 \%$ \\
1.0 & $10 \%-30 \%$ \\
1.5 & $30 \%-50 \%$ \\
2.0 & $50 \%-70 \%$ \\
2.5 & $70 \%-90 \%$ \\
3.0 & $>90 \%$ \\
\hline
\end{tabular}


HAS-LPs after nasal instillation. After adaptive feeding for 5 days, the mice were randomly divided into two groups $(\mathrm{n}=$ 50): the HAS-LP group and the HAS group. Afterwards, mice were subcutaneously injected with D-galactose (500 $\mathrm{mg} \mathrm{kg}^{-1}$ per mouse) daily for 42 consecutive days according to the previous modelling schedule and were treated with HAS or HAS-LP nasal drops at a dose of $5 \mathrm{mg} \mathrm{kg}^{-1}$. Blood samples were collected from mice in each group at $0.033,0.083,0.25,0.5,1,2,4,8,12$ and $24 \mathrm{~h}$, and brain tissue samples were collected after the mice were sacrificed.

Plasma samples were collected in heparin-containing microcentrifuge tubes, which were then centrifuged rapidly at $4000 \mathrm{rpm}$ for 10 minutes at $4^{\circ} \mathrm{C}$ to collect the supernatant and obtain the plasma samples. Before the quantitative detection of HAS levels in plasma, a methanol solution was added to the plasma samples (1.8 mL/0.6 mL plasma), vortexed for 3 minutes and then centrifuged at $12,000 \mathrm{rpm}$ for 10 minutes. The supernatant containing HAS was transferred to another tube and placed under nitrogen to remove methanol. The residue was reconstituted in $0.1 \mathrm{~mL}$ of methanol and centrifuged at $12,000 \mathrm{rpm}$ for $10 \mathrm{~min}$. Finally, $10 \mathrm{uL}$ of supernatant were removed and injected into the HPLC system for analysis. The HPLC method was the same as the aforementioned HAS liquid phase detection. The collected brain tissue samples were first milled in normal saline $(6 \mathrm{~mL} / 1$ $\mathrm{g}$ brain tissue) and then the operation was the same as that used for plasma samples.

The pharmacokinetic analysis was performed using a noncompartmental design and the pharmacokinetic parameters, including maximum concentration $\left(\mathrm{C}_{\max }\right)$ and the corresponding time (tmax), area under the concentrationtime curve (AUC), mean residence time (MRT), half life $\left(\mathrm{t}_{1 / 2}\right)$, and clearance (CL), were computed using DAS 2.0 software. In addition, the relative uptake rate (Re), drug targeting efficiency (DTE) and peak concentration ratio (Ce) were also calculated using the following equations:

$$
\begin{gathered}
\mathrm{Re}=\mathrm{AUC}_{H A S-L P} / \mathrm{AUC}_{H A S} \\
\mathrm{DTE}=\mathrm{TE}_{(H A S-L P)} / \mathrm{TE}_{(H A S)} \\
\mathrm{TE}=\mathrm{AUC}_{\text {brain }(0-\mathrm{t})} / \mathrm{AUC}_{\text {plasma }(0-\mathrm{t})} \\
\mathrm{Ce}=\mathrm{C}_{\text {max }- \text { brain }(H A S-L P)} / \mathrm{C}_{\text {max }- \text { brain }(H A S)}
\end{gathered}
$$

\section{Statistical Analysis}

Data are presented as the mean \pm standard deviations (SD). Statistical comparisons were performed using Student's $t$-test or one-way analysis of variance (ANOVA) with GraphPad Prism 5 software (GraphPad Software Inc., La Jolla, CA). $\mathrm{P}<0.05$ was considered significant.

\section{Results}

\section{Preparation of HAS-LPs}

As mentioned above, the chemical structure of HAS is extremely unstable and it is easily converted to its isomers under ultraviolet light and oxygen, eventually losing its pharmacological activity. Nanoliposomes have a phospholipid bilayer structure that can encapsulate drugs. To a certain extent, it can isolate drugs from ultraviolet light and oxygen, thus increasing the stability of the drug. At the same time, because the bimolecular structure of phospholipids is similar to the cell membrane, nanoliposomes also enable drugs to better overcome the bloodbrain barrier (BBB). Therefore, in this experiment, we considered encapsulating HAS into HAS-LPs, to improve drug stability and improve its curative effect. When HAS was encapsulated HAS-LPs, soy lecithin and cholesterol were chosen as lipid materials due to their nontoxic properties. In initial experiments, we found that the weight ratio of HAS to lipid material, the ratio of cholesterol to soy lecithin, and the volume of ultrapure water were factors significantly affecting the encapsulation efficiency and loading capacity of HAS-LPs. Therefore, three different levels of these three factors were selected and the interaction effect was analysed using $3^{3}$ factorial analyses. The results were shown in Table 3 , and indicated that the highest encapsulation efficiency and drug loading were obtained for treatment 2, when the mass ratio of HAS to lipids was $1: 20$, the mass ratio of cholesterol to soybean lecithin was $1: 4$, and the volume of ultrapure water was $15 \mathrm{~mL}$. Therefore, in subsequent experiments, we chose these conditions as the formulation to prepare HAS-LPs and continued to study the stability of HAS-LPs under conditions.

\section{Characterization, Storage Stability and Drug Release Rate of HAS-LP}

In this experiment, related characterization studies were conducted on the prepared HAS-LPs (the preparation process is shown in Figure 3A), which mainly included TEM, the particle size distribution, Zeta potential and release rate 


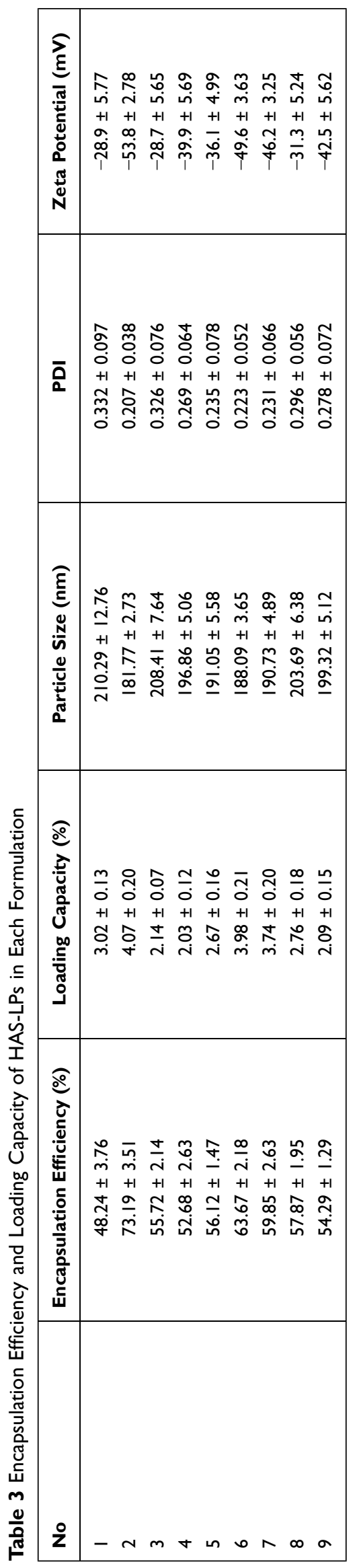

of HAS-LP. The results are described below. First, according to TEM observations, HAS-LPs had a spherical morphology and were uniformly dispersed (Figure 3B). Meanwhile, the particle size measurement results confirmed this conclusion. The average size distribution of HAS-LP was $181.77 \pm 2.73$ $\mathrm{nm}$ and the polydispersity index was $0.207 \pm 0.038$ (Figure 3C). In addition, the zeta potential was $-53.8 \pm$ $2.78 \mathrm{mV}$ (Figure 3D), indicating a high absolute negative zeta potential, good stability, and avoidance of particle aggregation. ${ }^{20}$

We stored HAS-LPs at $4^{\circ} \mathrm{C}$ for 8 weeks and $25^{\circ} \mathrm{C}$ for 1 week, and then continued to explore its particle size distribution, Zeta potential, and detected the content of HAS encapsulated in it to further verify their stability. After 8 weeks of storage, the average size distribution of HAS-LPs was 184.07 $\pm 2.96 \mathrm{~nm}$, the PDI was $0.216 \pm 0.055$ and the Zeta potential was $-57.5 \pm 3.02 \mathrm{mV}$, which was slightly different from the initially prepared HAS-LPs (Figure 3C and D). Moreover, after storage at $25^{\circ} \mathrm{C}$ for one week, the average size distribution of HAS-LP was $182.45 \pm 2.21 \mathrm{~nm}$, the PDI was $0.197 \pm$ 0.031 and the Zeta potential was $-51.1 \pm 3.21 \mathrm{mV}$ (Figure $3 \mathrm{C}$ and D). In addition, after storage at $25^{\circ} \mathrm{C}$ and 4 ${ }^{\circ} \mathrm{C}$, the content of free HAS decreased from $0.181 \pm 0.02 \mathrm{mg}$ $\mathrm{mL}^{-1}$ to $0.034 \pm 0.01 \mathrm{mg} \mathrm{mL}^{-1}$ and $0.096 \pm 0.02 \mathrm{mg} \mathrm{mL}^{-1}$, respectively. At the same time, the content of HAS contained in HAS-LP only changed from $0.181 \pm 0.02 \mathrm{mg} \mathrm{mL}^{-1}$ to $0.177 \pm 0.04 \mathrm{mg} \mathrm{mL}^{-1}$, when stored at $4^{\circ} \mathrm{C}$ for 8 weeks. The similar results were observed after storage at $25^{\circ} \mathrm{C}$ for 1 week, while the content of HAS contained in HAS-LP was $0.175 \pm 0.03 \mathrm{mg} \mathrm{mL}^{-1}$ (Figure 3E). Based on these results, HAS-LPs substantially inhibit the conversion of HAS.

The continuous release of drugs is an important parameter of the drug delivery system. Therefore, the release of free HAS and HAS-LPs was evaluated in PBS buffer (the testing process is shown in Figure 4A). As shown in Figure 4B, the release rate of free HAS was $83.82 \%$ at $2 \mathrm{~h}$ and was completely released at $8 \mathrm{~h}$, while HAS-LPs only released $56.89 \%$ at $2 \mathrm{~h}$, and only $74.72 \%$ of the drug was released at $8 \mathrm{~h}$. At 24 h, only $89.65 \%$ of the drug was released. Therefore, HASLPs control and delay the release of HAS. Moreover, we analyzed the release models of each group. The release mode of the HAS group resembled the first-order release model with an $R^{2}$ value of 0.9994 , while the release mode of the HAS-LP group was closer to the Higuchi release model with an $R^{2}$ value of 0.99046 (Figure 4C and D). These results provided evidence confirming that HAS-LPs not only slowed the release of HAS but also changed the release model. 
A

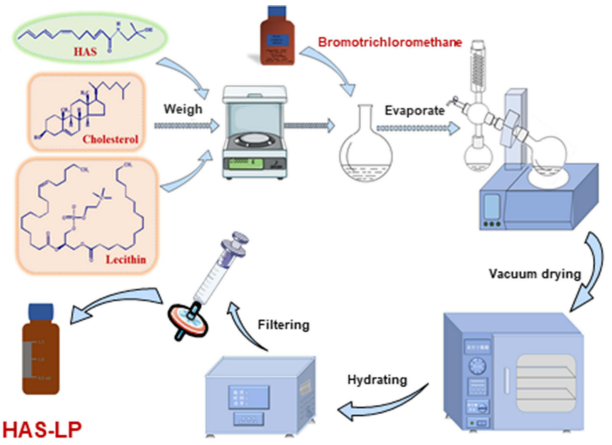

B

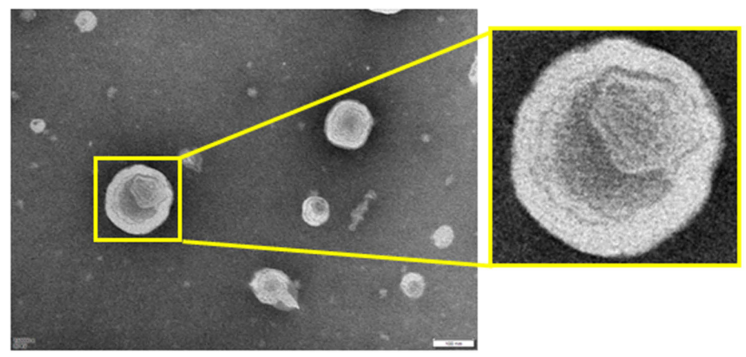

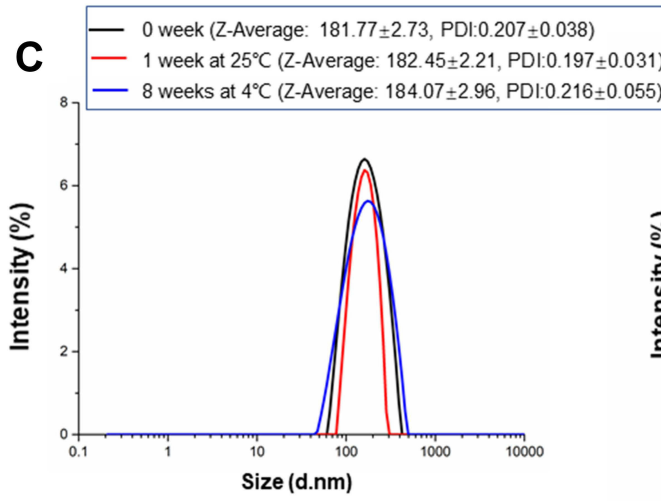
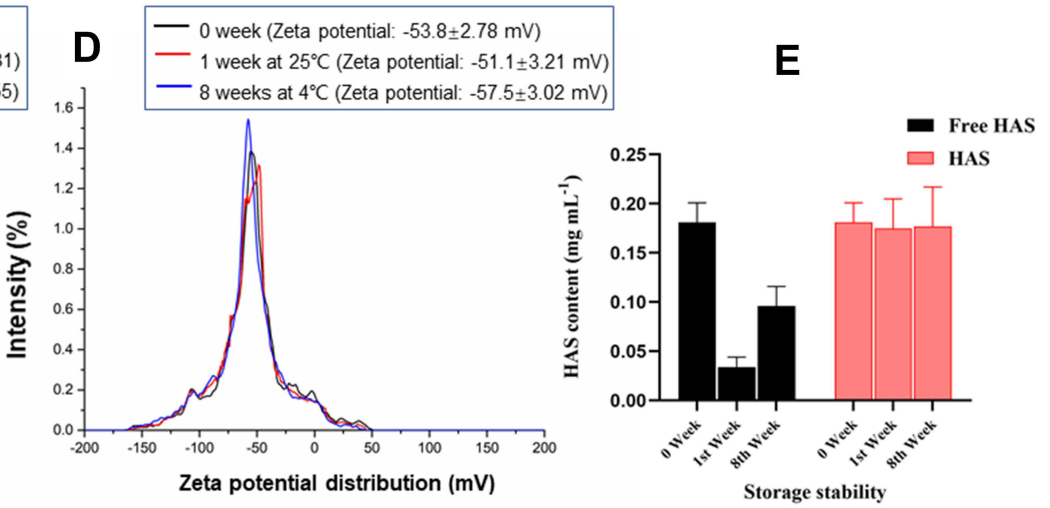

Figure 3 (A) Schematic illustrating the process used to prepare hydroxy- $\alpha$-sanshool nanoliposomes. (B) Morphology of HAS-LPs. (C) Size distribution of HAS-LPs after storage for $0,1\left(25^{\circ} \mathrm{C}\right)$ and $8\left(4^{\circ} \mathrm{C}\right)$ weeks. (D) Zeta potential of HAS-LPs after $0,1\left(25^{\circ} \mathrm{C}\right)$ and $8\left(4^{\circ} \mathrm{C}\right)$ weeks. (E) HAS contents in free HAS and HAS-LPs after storage at $25^{\circ} \mathrm{C}$ for I week and $4{ }^{\circ} \mathrm{C}$ for 8 weeks.

A

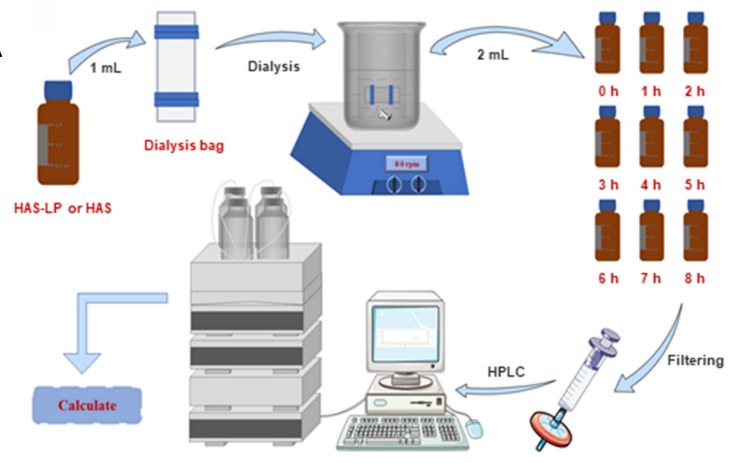

C

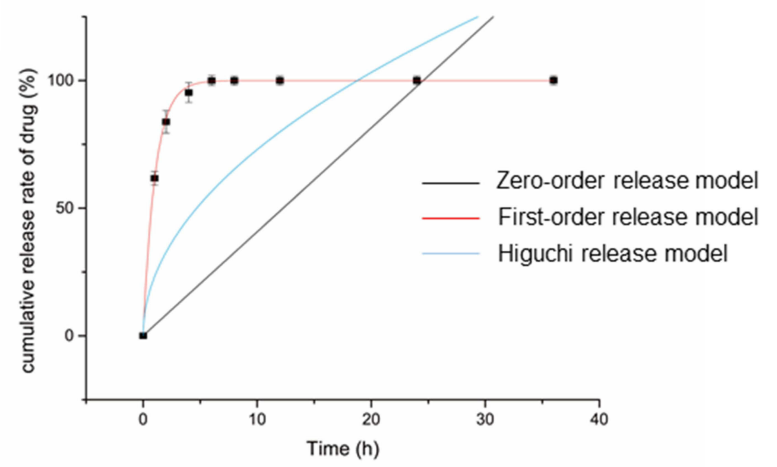

B

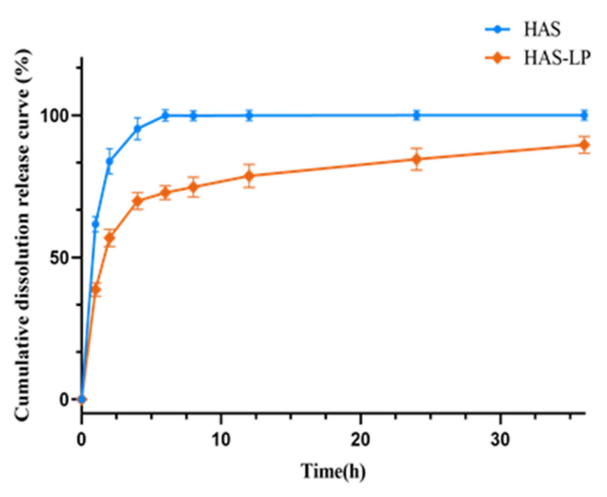

D

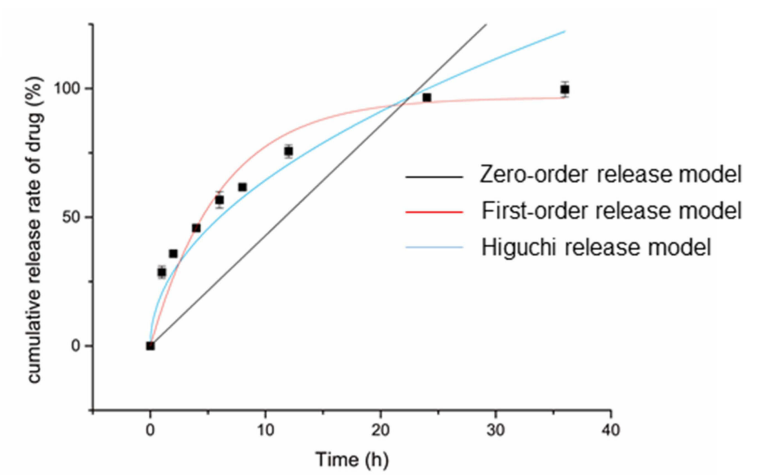

Figure 4 (A) Schematic illustration of the determination of hydroxy- $\alpha$-sanshool nano-liposomes release. (B) The continuous Release curve of HAS and HAS-LP in vitro. (C) Release model of the group of HAS. (D) Release model of the group of HAS-LP. 


\section{Results of the Nasal Mucosa Toxicity Investigation}

Figure 5 shows that after HE staining, the nasal mucosa in normal rats was still relatively complete and continuous without obvious lesions, while $1 \%$ deoxycholic acid nasal drops caused more serious damage to the nasal mucosa, including mucosal epithelia loss, lamina propria necrosis, inflammatory cell infiltration and vascular congestion in the lamina propria. At the same time, the nasal mucosa was slightly damaged in the HAS nasal drip group, as evidenced by a small amount of blood congestion in the lamina propria. However, in contrast, the HAS-LP nasal drip group showed almost no damage to the nasal mucosa and the findings were equivalent to that of the normal group, indicating that HASLP can be safely used for nasal administration.

\section{HAS-LP Nose Drops Ameliorated Cognitive Impairment in D-Galactose- Treated Mice}

The passive avoidance test is designed according to the animals' preference for darkness and aversion to light, and their fear memory of aversive stimuli such as foot electric shock. In other words, this design reflect the learning and memory abilities of animals to a certain extent. No significant differences in escape latency and error numbers were observed among the groups during training. However, in the testing trial, compared with the normal group, the escape latency of the model group was significantly shortened $(\mathrm{P}<0.01)$, and the number of errors was significantly increased (Figure 6, $\mathrm{P}<0.01$ ). Based on this result, the learning and memory abilities of mice were impaired after D-galactose treatment Furthermore, interestingly, after HAS-LP nasal drip treatment, the escape latency of the mice was significantly prolonged $(\mathrm{P}<0.01)$. Meanwhile, HAS-LP treatment at doses of 2.5 and $5.0 \mathrm{mg} \mathrm{kg}^{-1}$ obviously reduced the number of errors (Figure 6 , $\mathrm{P}<0.01)$. Both of these results indicated that the HAS-LP nasal drip improved the learning and memory abilities of the mice compared with the model group.

Subsequently, the Morris water maze test was used to evaluate the effect of HAS-LPs on D-galactose-induced impairments in spatial memory and long-term memory in mice (Figure 7). In the orientation navigation test, the escape latency of mice in the control group was significantly longer than that of the normal mice $(\mathrm{P}<0.01)$, and each HAS-LP administration group showed a shorter escape latency than the control group (Figure 7A, $\mathrm{P}<0.01$ ). Moreover, in the spatial search test, the mice in control group crossed the platform location fewer times and displayed a shorter residence time in the target quadrant, indicating an obvious spatial memory impairment (Figure 7B and $\mathrm{C}, \mathrm{P}<0.01$ ). Nevertheless, after treatment with different doses of HAS-LP, the number of times the mice crossed over the platform location increased and the residence time was prolonged compared with the control group (Figure 7B and $\mathrm{C}, \mathrm{P}<0.01$ ). Collectively, these results suggested that HAS-LP could ameliorates D-galactoseinduced learning and memory impairments in mice.

\section{HAS-LP Ameliorated D-Galactose- Induced Hippocampal Neuron Damage}

Cognitive function is closely related to the structure of hippocampus. ${ }^{21}$ We examined the effects of HAS on
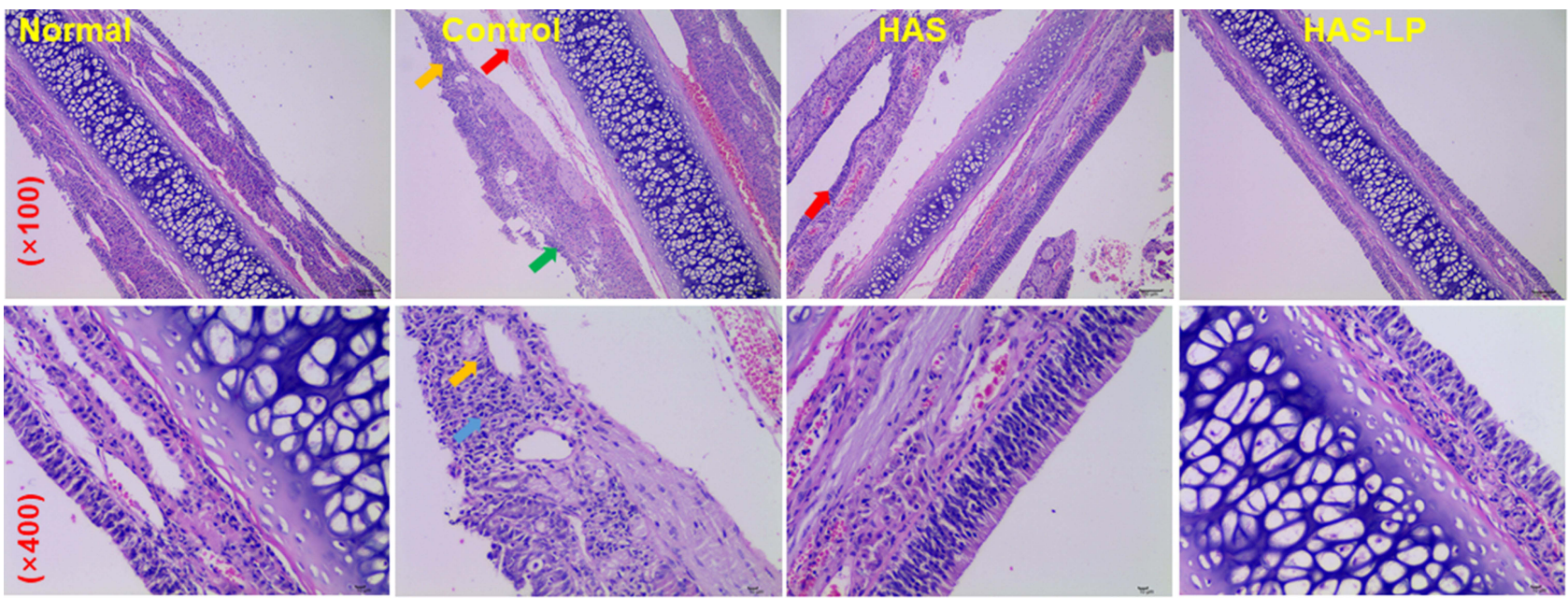

Figure 5 HE staining of nasal mucosa (the green arrow showed congestion of blood vessel, yellow arrow showed lamina propria necrosis, green arrow showed epithelial cell necrosis and blue arrow showed inflammatory cell infiltration). 

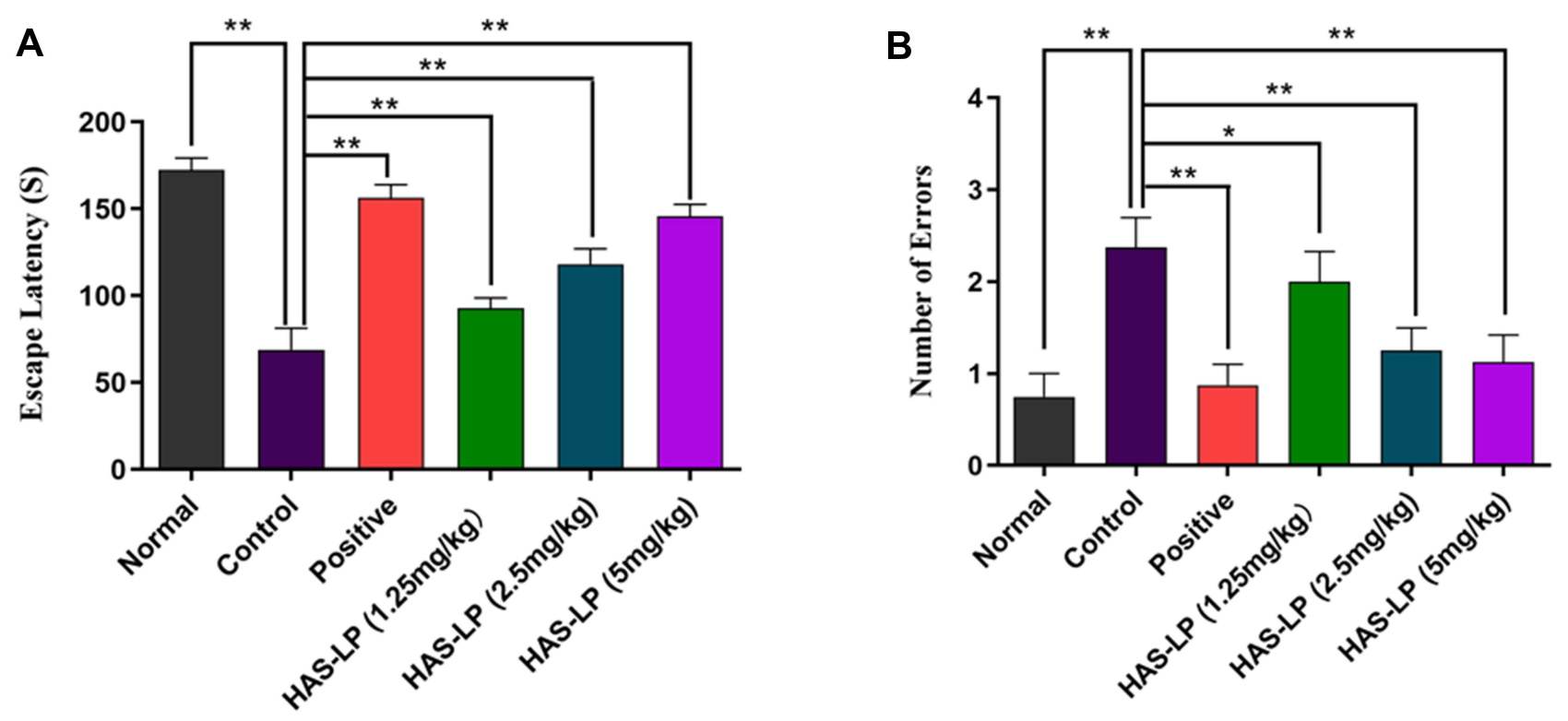

Figure 6 HAS-LP treatment attenuated learning and memory deficits in the passive avoidance test. (A) The escape latency. (B) The number of errors. Data are expressed as means \pm S.D. $(n=8)$. ${ }^{*} * \mathrm{P}<0.01$ vs the control group; $* \mathrm{P}<0.05$ vs the control group.

D-galactose induced hippocampal pathology in mice to further assess the effects of HAS-LP treatment on cognitive dysfunction. HE staining showed that the structure of hippocampal neurons in the normal group was complete, with clear boundaries between nucleus and cytoplasm, and cells were neatly arranged; no obvious cell degeneration or necrosis was observed, and damage index in CA1 and CA3 were low (Figure 8A). In contrast, the hippocampus of the control group showed pathological features such as atrophy, deformation, and loose arrangement of neurons in the hippocampus, and the damage indexes in CA1 and CA3 were both increased (Figure 8B, $\mathrm{P}<0.01$ ). At the same time, after treatment of different doses of HAS-LP, the pathological changes of the nerve cells in the CA1 and CA3 regions were significantly reversed, the damage indexes were reduced, and the efficacy of $5.0 \mathrm{mg} \mathrm{kg}^{-1}$ of HAS was similar to that of the positive group (Figure $8 \mathrm{~B}$ ). Nissl staining, a significantly lower number of Nissl bodies in the CAI and $\mathrm{CA} 3$ regions of hippocampus in the control group than in the normal group (Figure $8 \mathrm{~B}$, $\mathrm{P}<0.01$ ), and this phenomenon was alleviated to varying degrees after HAS treatment. In addition, Tunel staining was used to observe apoptotic cells in CA1 and CA3 regions, and the results showed that the percentage of apoptotic cells in the control group was significantly higher than that in the normal group, while HAS-LP reversed this phenomenon (Figure 9, $\mathrm{P}<0.01$ ). In summary, all these staining results indicated that D-galactose induced hippocampal nerve damage could be relieved by HAS-LP treatment.

\section{Pharmacokinetics of HAS-LP}

The potential for targeted delivery of HAS-LP in vivo was further evaluated. The temporal profiles of plasma and brain HAS concentrations are shown in Figure 10, and the pharmacokinetic parameters are summarized in Table 4. Nasally administered HAS and HAS-LPs were rapidly absorbed through nasal mucosa, as evidenced by high plasma drug concentrations at $0.5 \mathrm{~h} . \mathrm{T}_{1 / 2}$ of free HAS was $4.140 \pm 0.893 \mathrm{~h}$, while $T_{1 / 2}$ of HAS-LPs increased to $4.444 \pm 0.754 \mathrm{~h}$. In addition, the MRT of free HAS was $4.178 \pm 0.565 \mathrm{~h}$, while the value of HAS-LPs increased to $6.296 \pm 1.022 \mathrm{~h}$ and the CL of HAS-LP group decreased significantly from $0.842 \pm$ $0.178 \mathrm{~L} \mathrm{~kg}^{-1} \mathrm{~h}^{-1}$ to $0.56 \pm 0.206 \mathrm{~L} \mathrm{~kg}^{-1} \mathrm{~h}^{-1}$. Based on these results, HAS-LPs enabled the sustainable release of HAS. The relevant pharmacokinetic parameters were further observed, compared with free HAS, the area under the plasma concentration time curve (AUC) of HAS-LPs was increased 0.72-fold compared with that of HAS (8.795 \pm $1.428 \mathrm{mg} \mathrm{h} \mathrm{L}^{-1}$ vs $5.111 \pm 1.119 \mathrm{mg} \mathrm{h} \mathrm{L}^{-1}, \mathrm{p}<0.01$ ), and $\mathrm{C}_{\max }$ also increased from $1.398 \pm 0.289 \mathrm{mg} \mathrm{L}^{-1}$ to $1.906 \pm$ $0.343 \mathrm{mg} \mathrm{L}^{-1}$, indicating that HAS-LPs were more likely to be enriched in plasma than free HAS.

In addition, the related pharmacokinetic parameters of brain tissue were analyzed. The AUC of HAS-LPs in the brain was $14.414 \pm 2.811 \mathrm{mg} \mathrm{h}^{-1} \mathrm{~kg}^{-1}$, and the $\mathrm{C}_{\max }$ 
A

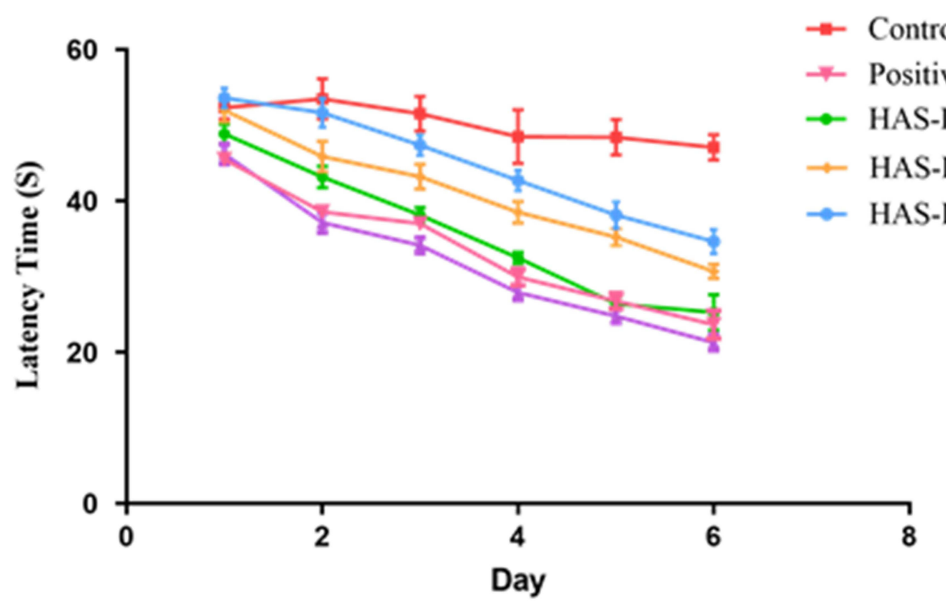

- Normal

ontrol

itive

-LP $(5 \mathrm{mg} / \mathrm{kg})$

S-LP $(2.5 \mathrm{mg} / \mathrm{kg})$
B

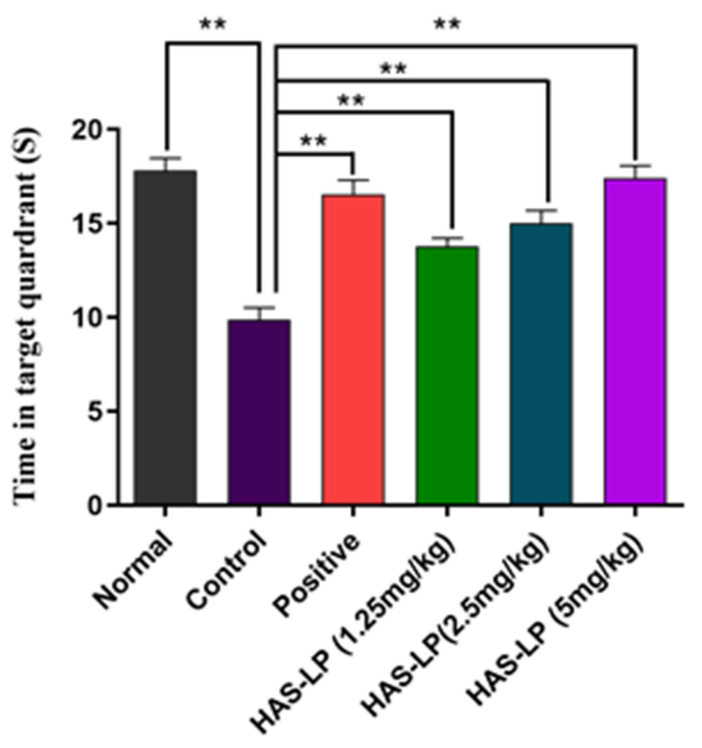

C

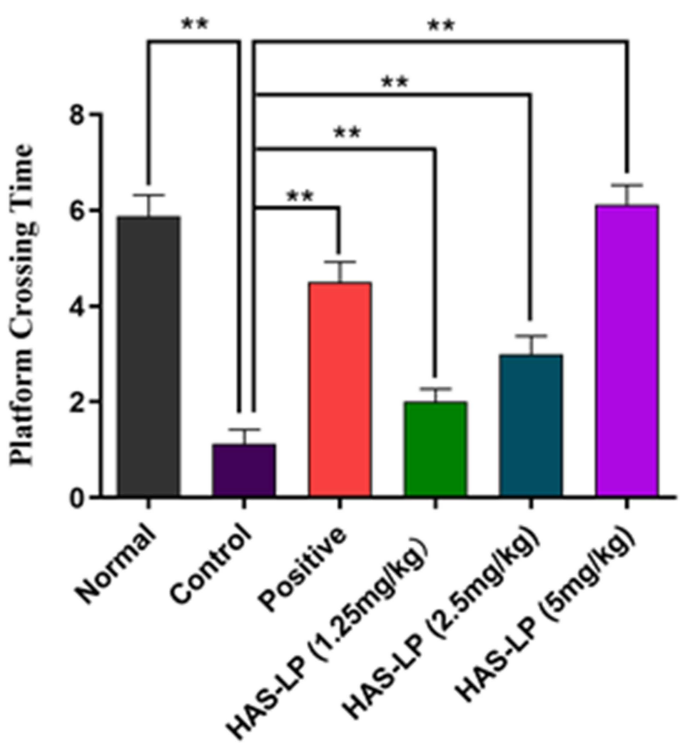

Figure 7 HAS-LP treatment attenuated learning and memory deficits in the Morris water maze test. (A) The mean escape latency time in the training trials. (B) The number of crossing platforms. (C) The residence time spent in the target quadrant. Data are expressed as means \pm S.D. $(n=8)$. **P $<0.0 \mathrm{I}$ vs the control group.

reached $3.015 \pm 1.305 \mathrm{mg} \mathrm{kg}^{-1}$, both of which were significantly greater than with the values of the HAS group $(\mathrm{P}<0.01$ and $\mathrm{P}<0.05$, respectively). These results indicated that HAS was more easily absorbed by the nasal mucosa after being encapsulated in liposomes to increase the concentration at target sites and facilitate nasal to brain transportation. Meanwhile, the $T_{1 / 2}$ of HAS-LP group was longer than that of the HAS group $(\mathrm{P}<0.05)$, and the MRT time was extended to $6.412 \pm 1.481 \mathrm{~h}$, implying the efficiency of HAS-LP in prolonging the retention time of HAS after entering the brain, which is conducive to the accumulation of drugs in the brain for a longer time and in greater amounts. After calculation, the Re of HASLP was 2.237, Ce was 1.154, and DTE was 1.263. In the targeted preparation, $R \mathrm{e}>1$ indicates that the drug is targeted in the organ, and the higher the DTE value is, the stronger the selectivity is. Therefore, these results showed that after HAS been made into the corresponding liposome, its targeting was higher than that of free HAS solution.

\section{Discussion}

In our previous studies, HAS ameliorated learning and memory impairments in mice and we provided a brief description of the mechanisms involved. ${ }^{17}$ However, because HAS is an unsaturated chain fatty acid amide containing abundant cis-conjugated double bonds, it is extremely unstable, making it highly susceptible to 

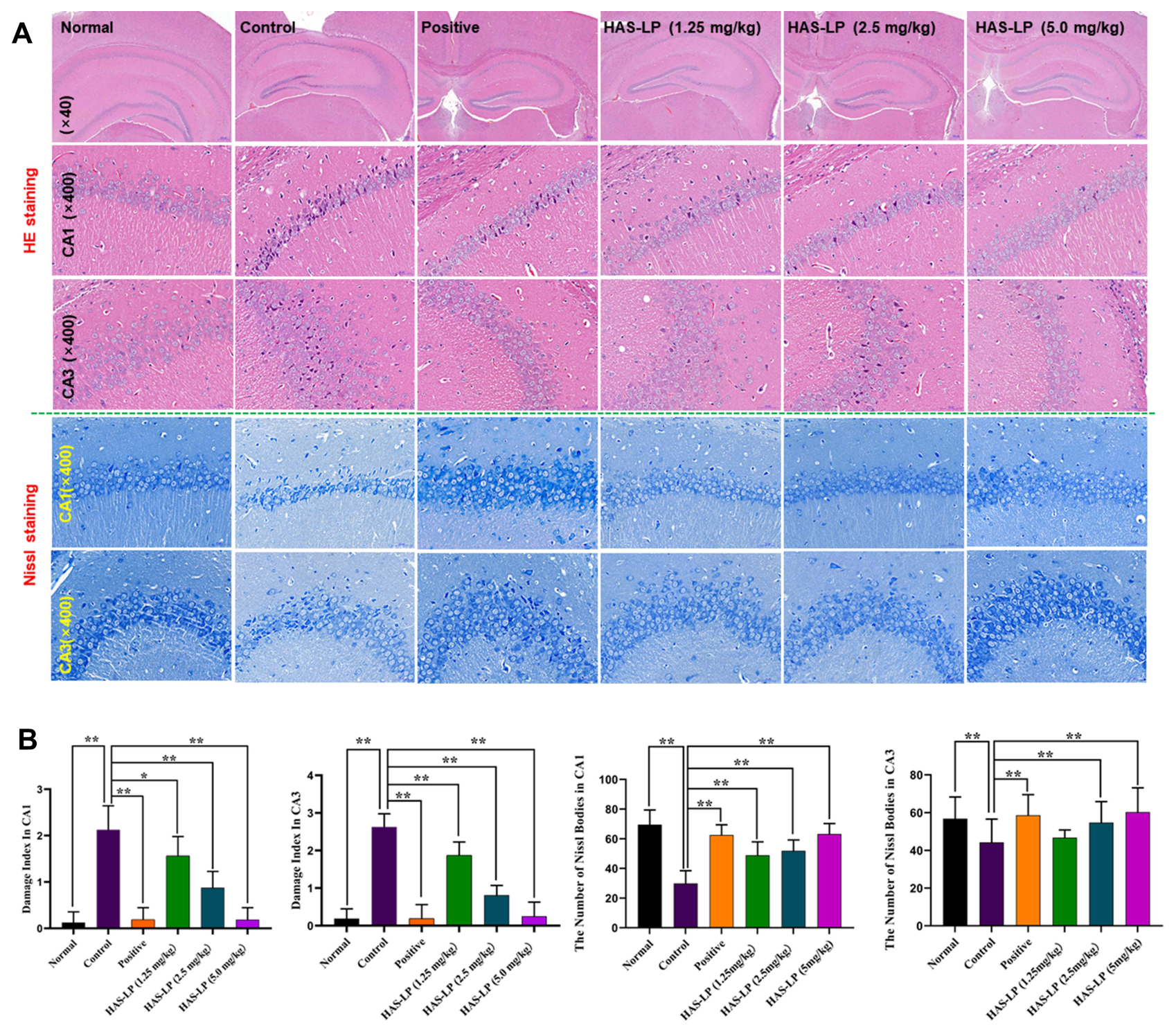

Figure 8 HAS-LP treatment attenuated hippocampal neuronal damage and loss. (A) Representative photomicrographs of HE staining and Nissl staining in the hippocampal CAI and CA3 regions are shown (magnification, 40x and 400x). (B) The damage index of D-galactose model mice (mean \pm SD, $n=5$ ) and the number of Nissl bodies of D-galactose model mice (mean $\pm \mathrm{SD}, \mathrm{n}=5$ ). $* \mathrm{P}<0.01$, **P $<0.05$ vs the control group.

chemical changes at room temperature or under UV light, which limits the efficacy and clinical application of HAS. Therefore, as part of our continuing investigation on HAS, we considered preparing various formulations of HAS to improve its stability.

A major obstacle in the treatment of $\mathrm{AD}$ is the inability of drug molecules to cross the $\mathrm{BBB}$, which prevents the drug from accumulating in the brain and fails to achieve effective drug concentrations and subsequent therapeutic effects. ${ }^{22,23}$ After considering all the factors, we decided to prepare HAS as a nanoliposome formulation. Liposomes are an emerging nanoformulation, and the encapsulation of a drug into liposomes can hide the drug in the phospholipid bilayer structure and improve its stability. ${ }^{24,25}$ In addition, encapsulating the drug in lipid material can also reduce the drug irritation, increase the slow release and the ability of the drug to be transported through the blood-brain barrier, thus enhancing the ability of the drug to target the brain. Therefore, incorporating HAS into liposomes will also potentially enable HAS to a better therapeutic effect on $\mathrm{AD}^{26-28}$ In the preparation of HAS into HAS-LPs, the film dispersion method was chosen and the encapsulation rate was used as a measurement target, which fully considered the lipid solubility of HAS and ensured that was it completely miscible with the lipid material and trichloromethane. In 
the subsequent process, ultrasonic hydration of the dispersed liposomes reduced the particle size of the formulation and improved the hydration efficiency. The HAS-LPs generated under the selected preparation conditions had small particle size, uniform dispersion, substantially improved stability compared to free HAS, and less difference in its apparent characteristics after 8 weeks at $4^{\circ} \mathrm{C}$. According to in vitro release assays, HAS-LP slowed the release of HAS and altered its release model, and gave HAS a slow release.

Nasal administration, a traditional mode of drug delivery, has recently been shown to transport drugs directly into the cerebrospinal fluid, which not only protects the integrity of the drug but also enables targeting. Therefore, in this study, transnasal administration of HAS-LP was considered to avoid the destruction of HAS by first-pass elimination during oral administration and to subsequently achieve improved bioavailability of HAS. In addition, the lipid material in HAS-LPs has a high structural similarity to the $\mathrm{BBB}$, which may also facilitate HAS transport across the $\mathrm{BBB}$ and is accumulation in the brain. ${ }^{29-31}$ In addition, a large number of nasal cilia are distributed in the nasal mucosa of animals, which constitute the first line of defence of the upper respiratory tract. Therefore, before administering a drug to the nasal mucosa, the nasal mucosal toxicity of the drug should first be examined, and then the animal
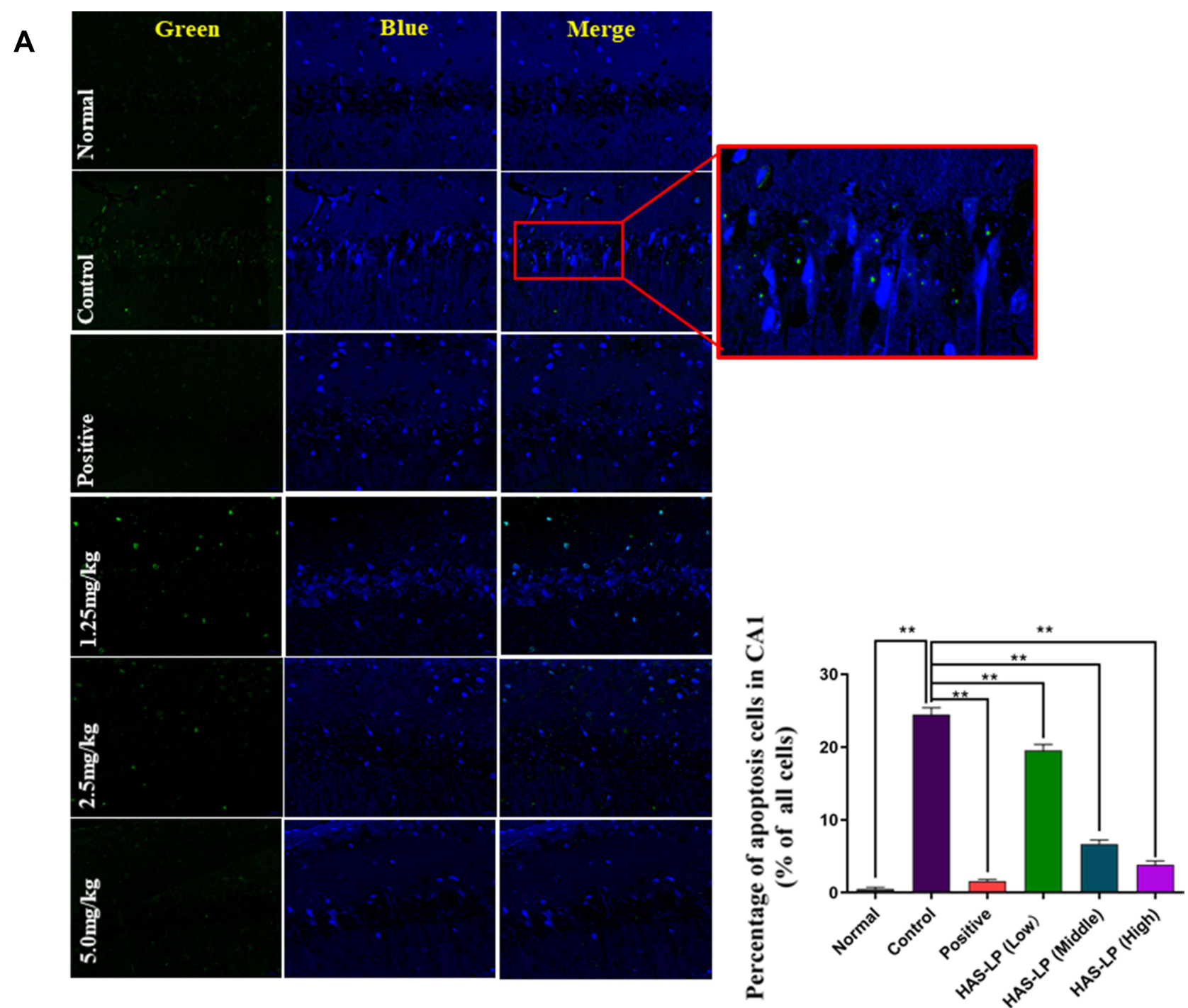

Figure 9 Continued. 


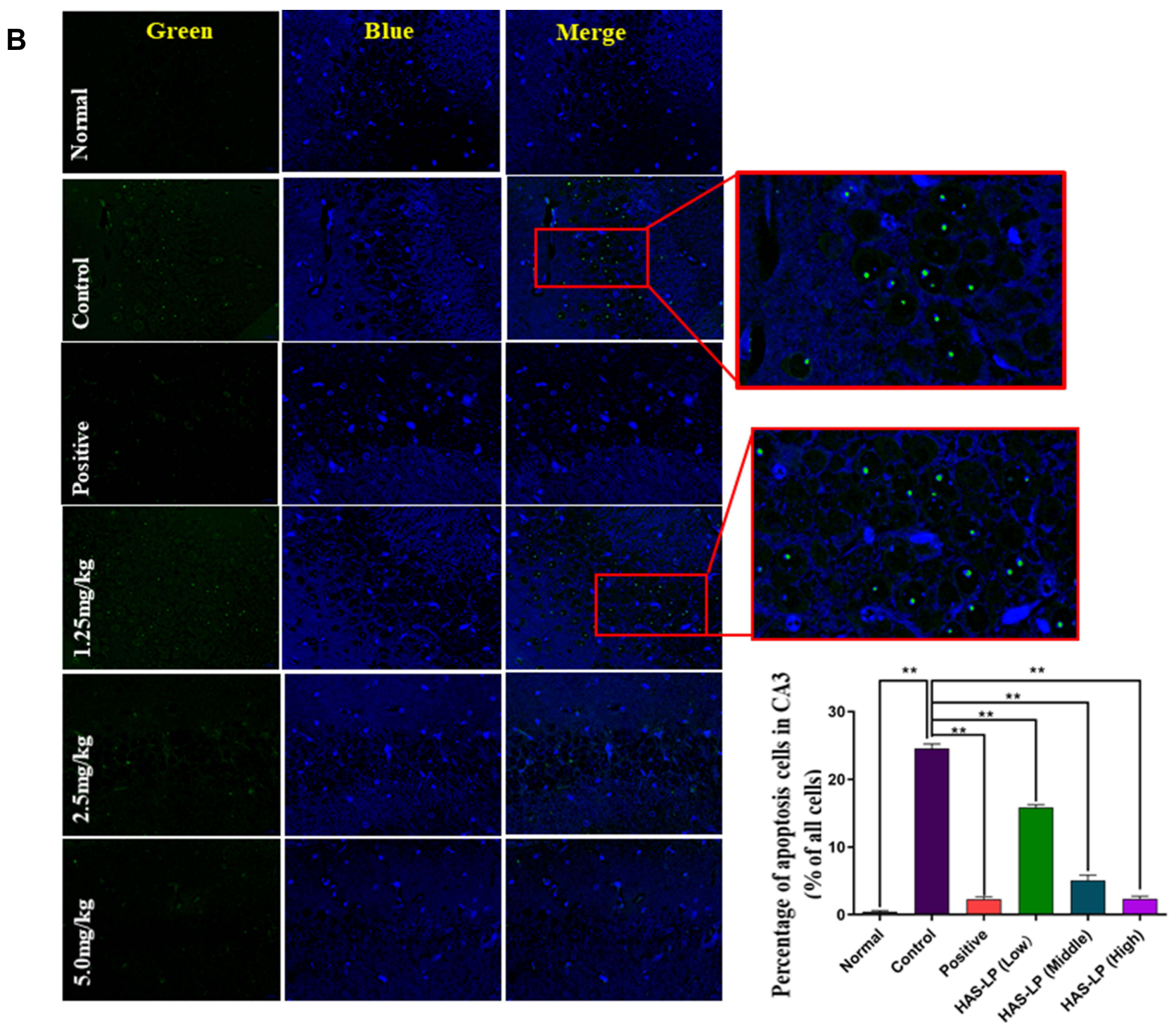

Figure 9 (A) Representative photomicrographs of Tunel staining in the hippocampal CAI regions (magnification, 400x) and the percentage of apoptotic cells in the CAI region of $D$-galactose model mice (mean $\pm S D, n=5$ ) are shown. (B) Representative photomicrographs of Tunel staining in the hippocampal CA3 regions and the percentage of apoptotic cells in the CA3 region of $D$-galactose model mice (mean $\pm S D, n=5$ ) are shown (magnification, $400 \times$ ). **P $<0.05$ vs the control group.

should be safely modelled and the drug administered. However, the unique nature of HAS irritates the nasal mucosa and can lead to pathological features such as congestion of the nasal mucosa. Not surprisingly, in our study, HAS encapsulated by lipid material solves this problem perfectly, as it did not cause any significant damage to the nasal mucosa after intranasal administration and was safe for nasal administration in subsequent experiments.

According to previous studies, AD is often accompanied by cognitive decline. ${ }^{32} \mathrm{D}$-galactose, a reducing sugar widely present in food and humans, is excreted from the body autonomously at low doses. ${ }^{33}$ However, when D-galactose is present in the body at high doses, it is converted to aldose and hydrogen peroxide through a reaction catalysed by galactose oxidase, which in turn leads to the excess production and accumulation of reactive oxygen species in the body, inducing a series of responses such as oxidative stress and cognitive dysfunction. ${ }^{34-36}$ Therefore, the mouse D-galactoseinduced damage model is often used to screen drugs for the treatment of cognitive dysfunction in $\mathrm{AD} .{ }^{37}$ In the present experiment, we used a mouse D-galactose- 

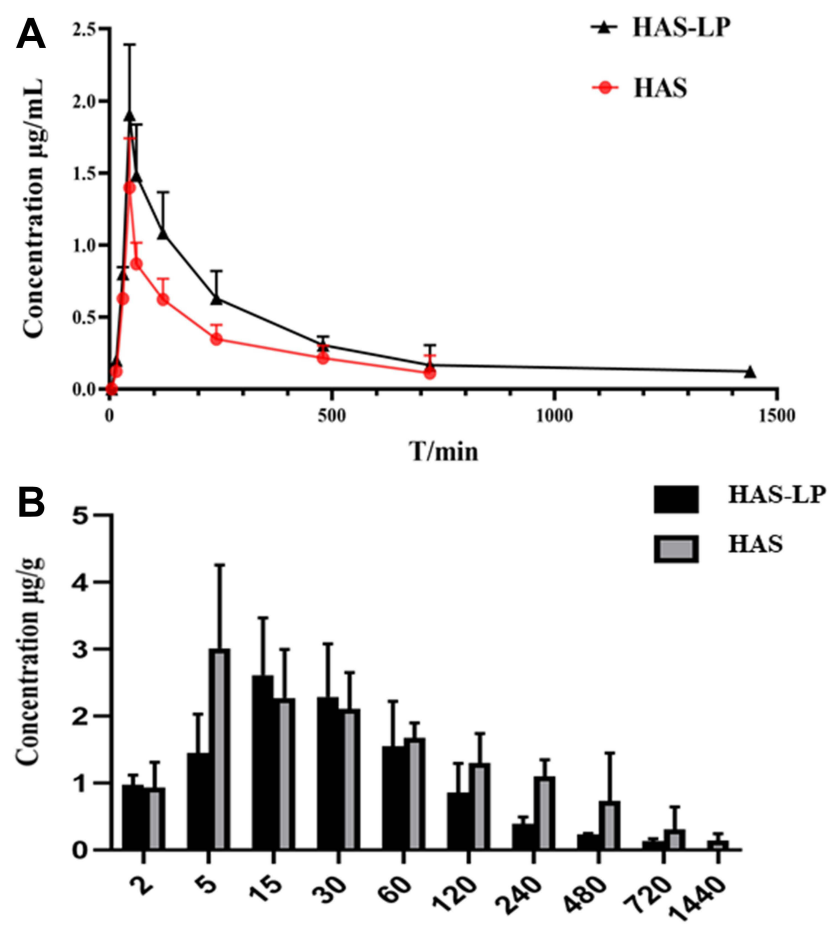

$\mathbf{T} / \mathbf{m i n}$

Figure 10 (A) Time profile of the plasma HAS level of mice after they were nasally administered with free HAS and HAS-LP. (B) Time profile of the brain tissue HAS level of mice after they were nasally administered with free HAS and HAS-LP.

induced damage model, and verified the effect of HAS-LP administration on ameliorating learning and memory impairments in mice using Morris water maze experiment and passive avoidance experiment. And then, among the pathological tests, we found that HAS-LPs protected neuronal cells in the CA1 and CA3 regions of the mouse hippocampus and inhibited apoptosis by performing $\mathrm{HE}$ staining, Nissl staining and TUNEL staining. Notably, in the pharmacokinetic studies, we compared free HAS with HAS-LPs. HAS-LPs was more highly enriched in brain tissues and plasma than the monomeric drug HAS, and the slow drug-release from HAS-LPs allowed the drug to exert a long-lasting effect in vivo and display some targeting properties.

\section{Conclusion}

In this study, HAS-LPs were prepared using the film dispersion method, while the mass ratio of HAS:cholesterol:soybean lecithin was $1: 4: 16$ and the amount of ultrapure water was $15 \mathrm{~mL}$ HAS-LPs had a uniform particle size and homogeneous dispersion, with strong stability and sustained release ability, and the release model was consistent with the Higuchi model. When HAS-LPs were applied nasally, they had no significant toxicity toward the nasal mucosa. Behavioral experiments were performed on D-galactose-induced ageing mice mainly using passive avoidance and water maze experiments. After nasal treatment with different doses of HAS-LPs in model mice, the learning and memory abilities were improved, which preliminarily suggested that HAS-LPs improved the learning and memory impairments in AD mice after transnasal administration. In the pathological assay, we likewise found that HASLPs protected neuronal cells from damage. Compared with free HAS, HAS-LPs are more easily enriched in plasma and brain tissue and have brain-targeting properties. In conclusion, HAS-LPs have potential value in improving the stability of HAS and the application to treat learning and memory deficits in individuals with AD.

Table 4 Pharmacokinetic Parameters of Free HAS and HAS-LP in Plasma and Brain Tissue ( $n=5$, Mean \pm SD) **P $<0.0$ I vs HAS Group; *P < 0.05 vs HAS Group

\begin{tabular}{|l|c|c|c|c|}
\hline \multirow{2}{*}{$\begin{array}{l}\text { Pharmacokinetic } \\
\text { Prameters }\end{array}$} & HAS & HAS-LP & HAS & HAS \\
\cline { 2 - 5 } & $5.11 \mathrm{II} \pm 1.199$ & $8.795 \pm 1.428^{* *}$ & $7.438 \pm 1.735$ & $15.787 \pm 2.854^{* *}$ \\
\hline $\mathrm{AUC}_{0-\mathrm{t}}\left(\mathrm{mg} \mathrm{h}^{-1} \mathrm{~L}^{-1}\right)$ & $4.178 \pm 0.565$ & $6.296 \pm 1.022^{* *}$ & $3.074 \pm 0.618$ & $6.412 \pm 1.48 I^{* *}$ \\
$\mathrm{MRT}_{0-\mathrm{t}}(\mathrm{h})$ & $4.140 \pm 0.893$ & $4.444 \pm 0.754$ & $5.116 \pm 0.729$ & $6.734 \pm 1.01 I^{*}$ \\
$t_{1 / 2}(\mathrm{~h})$ & $0.500 \pm 0.194$ & $0.500 \pm 0.052$ & $0.250 \pm 0.059$ & $0.083 \pm 0.030$ \\
$\mathrm{t}_{\max }(\mathrm{h})$ & $0.842 \pm 0.178$ & $0.56 \pm 0.206^{*}$ & None & None \\
$\mathrm{Cl}\left(\mathrm{L} \mathrm{h}^{-1} \mathrm{~kg}^{-1}\right)$ & $1.398 \pm 0.289$ & $1.906 \pm 0.343^{*}$ & $2.613 \pm 0.457$ & $3.015 \pm 1.305^{*}$ \\
$\mathrm{C}_{\max }\left(\mathrm{mg} \mathrm{L}^{-1}\right)$ & & & & \\
\hline
\end{tabular}




\section{Disclosure}

The authors report no conflicts of interest in this work.

\section{References}

1. Feigin VL, Nichols E, Alam T. Global, regional, and national burden of neurological disorders, 1990-2016: a systematic analysis for the Global Burden of Disease Study 2016. Lancet Neurol. 2019;18:459-480. doi:10.1016/S1474-4422(18)30499-X

2. Ege D. Action mechanisms of curcumin in Alzheimer's disease and its brain targeted delivery. Materials. 2021;14:3332. doi:10.3390/ ma14123332

3. Huang Y, Mucke L. Alzheimer mechanisms and therapeutic strategies. Cell. 2012;148:1204-1222. doi:10.1016/j.cell.2012.02.040

4. Livingston G, Barber J, Rapaport P, et al. START (STrAtegies for RelaTives) study: a pragmatic randomised controlled trial to determine the clinical effectiveness and cost-effectiveness of a manual-based coping strategy programme in promoting the mental health of carers of people with dementia. Health Technol Assess. 2014;18:1-242. doi:10.3310/hta18610

5. Catafau AM, Bullich S. Amyloid PET imaging: applications beyond Alzheimer's disease. Clin Transl Imaging. 2015;3:39-55. doi:10.1007/s40336-014-0098-3

6. Tramutola A, Lanzillotta C, Perluigi M. Oxidative stress, protein modification and Alzheimer disease. Brain Res Bull. 2017;133:88-96. doi:10.1016/j.brainresbull.2016.06.005

7. Miya MS, Tamargo IA, Abubakar MB, Kamal MA, Greig NH, Gan SH. The role of microRNAs in Alzheimer's disease and their therapeutic potentials. Genes. 2018;9:174. doi:10.3390/genes9040174

8. Pasha S, Gupta K. Various drug delivery approaches to the central nervous system. Expert Opin Drug Deliv. 2010;7:113-135. doi:10.1517/17425240903405581

9. Mohammad F, Sanjula B, Sahni JK, Ali J. Nanotherapeutics for Alzheimer's disease (AD): past, present and future. J Drug Target. 2012;20:97-113. doi:10.3109/1061186X.2011.607499

10. Long Y, Yang Q, Xiang Y, et al. Nose to brain drug delivery-A promising strategy for active components from herbal medicine for treating cerebral ischemia reperfusion. Pharmacol Res. 2020;159:104795. doi:10.1016/j.phrs.2020.104795

11. Agrawal M, Saraf S, Saraf S, et al. Nose-to-brain drug delivery: an update on clinical challenges and progress towards approval of anti-Alzheimer drugs. $J$ Control Release. 2018;281:139-177. doi:10.1016/j.jconrel.2018.05.011

12. Joshi S, Singh-Moon RP, Wang M, et al. Transient cerebral hypoperfusion assisted intraarterial cationic liposome delivery to brain tissue. J Neuro-Oncol. 2014;118:73-82. doi:10.1007/s11060-014-1421-6

13. Markoutsa E, Papadia K, Giannou AD, et al. Mono and dually decorated nanoliposomes for brain targeting, in vitro and in vivo studies. Pharm Res. 2014;31:1275-1289. doi:10.1007/s11095-013-1249-3

14. Xiang L, Liu Y, Xie C, et al. The chemical and genetic characteristics of Szechuan pepper (Zanthoxylum bungeanum and Z. armatum) cultivars and their suitable habitat. Front Plant Sci. 2016;7:467. doi:10.3389/fpls.2016.00467

15. Lin Z, Han S, Jiang J, et al. Antitumor compound identification from Zanthoxylum bungeanum essential oil based on composition-activity relationship. Chem Res Chin Uni. 2013;6:1068-1071. doi:10.1007/ s40242-013-3223-3

16. Zhu RX, Zeng WC, Zhao ZF. Chemical components and antibacterial activity of Hanyuan Zanthoxylum bungeanum seed oil. Food Sci. 2011;17:85-88. doi:10.1097/RLU.0b013e3181f49ac7

17. Li RL, Zhang Q, Liu J, et al. Hydroxy- $\alpha$-sanshool possesses protective potentials on $\mathrm{H}_{2} \mathrm{O}_{2}$-Stimulated $\mathrm{PC} 12$ cells by suppression of oxidative stress-induced apoptosis through regulation of PI3K/Akt signal pathway. Oxid Med Cell Longev. 2020;2020:3481758. doi:10.1155/2020/3481758
18. Fsa B, Se A, Gma B, et al. Physical and cognitive training attenuate hippocampal ischemia-induced memory impairments in rat. Brain Res Bull. 2020;155:202-210. doi:10.1016/j.brainresbull.2019.10.007

19. Zhang MM, Xie MG, Wei DN, et al. Hydroxy- $\alpha$-sanshool isolated from Zanthoxylum bungeanum attenuates learning and memory impairments in scopolamine-treated mice. Food Funct. 2019;10:7315-7324. doi:10.1039/c9fo00045c

20. Luo R, Lin M, Fu C, et al. Calcium pectinate and hyaluronic acid modified lactoferrin nanoparticles loaded Rhein with dual-targeting for ulcerative colitis treatment. Carbohydr Polym. 2021;263:117998. doi:10.1016/j.carbpol.2021.117998

21. Zhao MH, Tang XQ, Gong D. Bungeanum improves cognitive dysfunction and neurological deficits in D-galactose-induced aging mice activating PI3K/Akt/Nrf2 signaling pathway. Front Pharmacol. 2020;11:71. doi:10.3389/fphar.2020.00071

22. Zhang X, Wang R, Hu D, et al. Oligodendroglial glycolytic stress triggers inflammasome activation and neuropathology in Alzheimer's disease. Sci Adv. 2020;6:eabb8680. doi:10.1126/ sciadv.abb8680

23. Gallagher M, Okonkwo OC, Resnick SM, et al. What are the threats to successful brain and cognitive aging? Neurobiol Aging. 2019;83:130-134. doi:10.1016/j.neurobiolaging.2019.04.016

24. Chen ZX. Research progress of Chinese medicine liposomes. J Pract Tradit Chin Med. 2010;26:587-590. doi:10.3760/cma.j.cn11214420200627-00372

25. Zhao Y, Tang G, Tang Q, et al. A method of effectively improved alpha-Mangostin bioavailability. Eur J Drug Metab Pharmacokinet. 2016;41:605-613. doi:10.1007/s13318-015-0283-4

26. Katdare A, Khunt D, Thakkar S. Comparative evaluation of fish oil and butter oil in modulating delivery of galantamine hydrobromide to brain via intranasal route: pharmacokinetic and oxidative stress studies. Drug Deliv Transl Res. 2020;10:1136-1146. doi:10.1007/ s13346-020-00739-y

27. Gou SQ, Huang YM, Wan Y, et al. Multi-bioresponsive silk fibroin-based nanoparticles with on-demand cytoplasmic drug release capacity for CD44-targeted alleviation of ulcerative colitis. Biomaterials. 2019;212:39-54. doi:10.1016/j.biomaterials.2019.05.012

28. Wang PQ, Liu Q, Xu WJ. Pure mechanistic analysis of additive neuroprotective effects between BA and jasminoidin in ischemic stroke mice. Acta Pharmacol Sin. 2018;39:961-974. doi:10.1038/ aps.2017.145

29. Gupta P, Bhattacharjee S, Sharma AR, Sharma G, Lee SS, Chakraborty C. miRNAs in Alzheimer disease - A therapeutic perspective. Curr Alzheimer Res. 2017;14:1198-1206. doi:10.2174/ 1567205014666170829101016

30. Ayers D, Scerri C. Non-coding RNA influences in dementia. Noncoding RNA Res. 2018;3:188-194. doi:10.1016/j. ncrna.2018.09.002

31. Carter SF, Herholz K, Rosa-Neto P, Pellerin LA. Astrocyte biomarkers in alzheimer's disease. Trends Mol Med. 2019;25:77-95. doi:10.1016/j.molmed.2018.11.006

32. Zhang B, Gaiteri C, Bodea LG, Wang Z, McElwee J, Podtelezhnikov AA. Integrated systems approach identifies genetic nodes and networks in late-onset Alzheimer's disease. Cell. 2013;153:707-720. doi:10.1016/j.cell.2013.03.030

33. Oskouei Z, Mehri S, Kalalinia F, Hosseinzadeh H. Evaluation of the effect of thymoquinone in d-galactose-induced memory impairments in rats: role of MAPK, oxidative stress, and neuroinflammation pathways and telomere length. Phytother Res. 2020;2252-2266. doi:10.1002/ptr.6982

34. Ullah F, Ali T, Ullah N, Kim MO. Caffeine prevents d-galactoseinduced cognitive deficits, oxidative stress, neuroinflammation and neurodegeneration in the adult rat brain. Neurochem Int. 2015;90:114-124. doi:10.1016/j.neuint.2015.07.001 
35. Wang LL, Huang Y, Wang G, Chen SD. The potential role of microRNA146 in 837 Alzheimer's disease: biomarker or therapeutic target? Med Hypotheses. 2012;78:398-401. doi:10.1016/j.mehy.2011.11.019

36. Acosta PB, Gross KC. Hidden sources of galactose in the environment. Eur $J$ Pediatr. 1995;154(7 Suppl 2):S87-S92. doi:10.1007/BF02143811
37. Wang Y, Zhang T, Zhao H. $\alpha$-pentoxifylline enhances antioxidative capability and promotes mitochondrial biogenesis in D-Galactoseinduced aging mice by increasing Nrf2 and PGC-1 through the cAMP-CREB pathway. Oxid Med Cell Longev. 2021;2021:6695613. doi:10.1155/2021/6695613

\section{Publish your work in this journal}

The International Journal of Nanomedicine is an international, peerreviewed journal focusing on the application of nanotechnology in diagnostics, therapeutics, and drug delivery systems throughout the biomedical field. This journal is indexed on PubMed Central, MedLine, CAS, SciSearch ${ }^{\mathbb{B}}$, Current Contents ${ }^{\mathbb{B}} /$ Clinical Medicine, $^{2}$
Journal Citation Reports/Science Edition, EMBase, Scopus and the Elsevier Bibliographic databases. The manuscript management system is completely online and includes a very quick and fair peer-review system, which is all easy to use. Visit http://www.dovepress.com/ testimonials.php to read real quotes from published authors. 